\title{
The genome sequence of the clouded yellow, Colias crocea
}

\section{(Geoffroy, 1785) [version 1; peer review: 2 approved]}

\author{
Sam Ebdon (D1), Alex Mackintosh1, Alex Hayward2, Karl Wotton (iD2, \\ Darwin Tree of Life Barcoding collective, \\ Wellcome Sanger Institute Tree of Life programme, \\ Wellcome Sanger Institute Scientific Operations: DNA Pipelines collective, \\ Tree of Life Core Informatics collective, Darwin Tree of Life Consortium
}

\footnotetext{
${ }^{1}$ Institute of Evolutionary Biology, University of Edinburgh, Edinburgh, UK

2University of Exeter, Penryn, UK
}

\section{V1 First published: 22 Oct 2021, 6:284 \\ https://doi.org/10.12688/wellcomeopenres.17292.1 \\ Latest published: 22 Oct 2021, 6:284 \\ https://doi.org/10.12688/wellcomeopenres.17292.1}

\begin{abstract}
We present a genome assembly from an individual female Colias crocea (also known as Colias croceus; the clouded yellow; Arthropoda; Insecta; Lepidoptera; Pieridae). The genome sequence is 325 megabases in span. The complete assembly is scaffolded into 32 chromosomal pseudomolecules, with the $\mathrm{W}$ and $\mathrm{Z}$ sex chromosome assembled. Gene annotation of this assembly on Ensembl has identified 13,803 protein coding genes.
\end{abstract}

Keywords

Colias crocea, Colias croceus, clouded yellow, genome sequence, chromosomal

This article is included in the Tree of Life gateway.

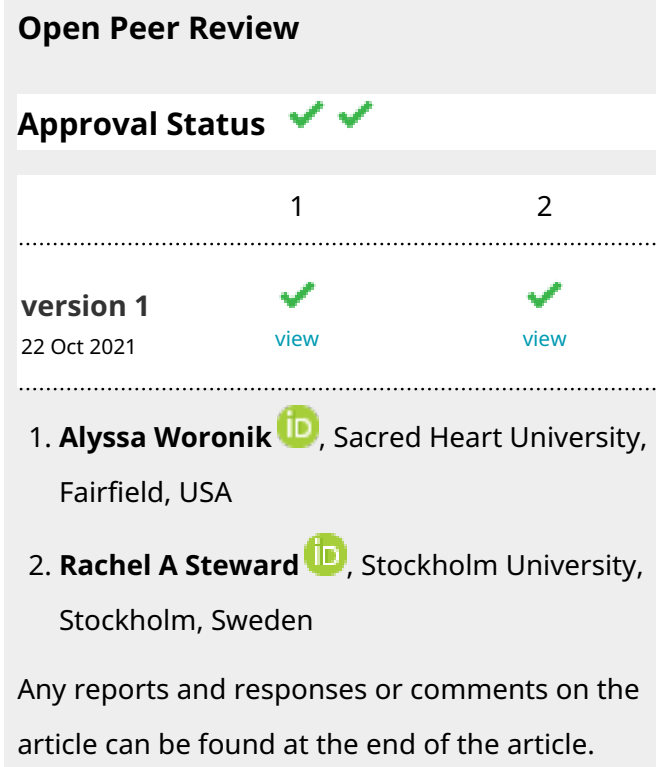

1

2

version 1

22 Oct 2021

view

1. Alyssa Woronik (D), Sacred Heart University, Fairfield, USA

2. Rachel A Steward ID, Stockholm University,

Stockholm, Sweden

Any reports and responses or comments on the article can be found at the end of the article. 
Corresponding author: Darwin Tree of Life Consortium (mark.blaxter@sanger.ac.uk)

Author roles: Ebdon S: Investigation, Resources, Visualization, Writing - Original Draft Preparation, Writing - Review \& Editing; Mackintosh A: Investigation, Resources; Hayward A: Investigation, Resources; Wotton K: Investigation, Resources;

Competing interests: No competing interests were disclosed.

Grant information: This work was supported by Wellcome through core funding to the Wellcome Sanger Institute (206194) and the Darwin Tree of Life Discretionary Award (218328). Fieldwork was supported by an ERC grant (ModelGenom Land 757648). Permissions for field sampling were obtained from the Gobierno de Aragon (INAGA/500201/24/2018/0614 to Karl Wotton). The funders had no role in study design, data collection and analysis, decision to publish, or preparation of the manuscript.

Copyright: (c) 2021 Ebdon S et al. This is an open access article distributed under the terms of the Creative Commons Attribution License, which permits unrestricted use, distribution, and reproduction in any medium, provided the original work is properly cited.

How to cite this article: Ebdon S, Mackintosh A, Hayward A et al. The genome sequence of the clouded yellow, Colias crocea (Geoffroy, 1785) [version 1; peer review: 2 approved] Wellcome Open Research 2021, 6:284

https://doi.org/10.12688/wellcomeopenres.17292.1

First published: 22 Oct 2021, 6:284 https://doi.org/10.12688/wellcomeopenres.17292.1 


\section{Species taxonomy}

Eukaryota; Metazoa; Ecdysozoa; Arthropoda; Hexapoda; Insecta; Pterygota; Neoptera; Endopterygota; Lepidoptera; Glossata; Ditrysia; Papilionoidea; Pieridae; Coliadinae; Colias; Colias crocea (also known as Colias croceus) (Geoffroy, 1785) (NCBI:txid72248).

\section{Background}

Colias crocea (or croceus), the clouded yellow, is a butterfly found in Europe, the middle east, and north Africa. This continuously-brooded migratory species visits the UK in the end of spring and summer, supplementing a small breeding population in the south. The larvae feed on a wide variety of leguminous plants, such as clovers (Trifolium sp.), alfalfa (Medicago sativa) and vetches (Vicia sp.). Despite recent declines, C. crocea has seen a large increase in both abundance and occurrence in the last 50 years in the British Isles (Fox et al., 2015) and is listed as Least Concern in the IUCN Red List (Europe) (van Swaay et al., 2010). A white polymorphism known as Alba (form helice) is associated with an alternative life-history strategy, where females reallocate wing pigment resources to somatic and reproductive development. This is associated with the insertion of a transposable element downstream of the homeobox transcription factor BarH-1 (Woronik et al., 2019). Colias crocea has 31 pairs of chromosomes, a genome size of approximately $318.6 \mathrm{Mb}$ (Woronik et al., 2019), and is female heterogametic (WZ). We note the recent production of a high-quality genome assembly for $C$. crocea (Woronik et al., 2019), and believe the sequence described here, generated as part of the Darwin Tree of Life project, will further aid understanding of the biology and ecology of this butterfly.

\section{Genome sequence report}

The genome was sequenced from a single female $C$. crocea collected from Bujaruelo, Aragon, Spain (latitude 42.7, longitude -0.1) (Figure 1). A total of 68-fold coverage in Pacific Biosciences single-molecule long reads and 91-fold coverage in 10X Genomics read clouds were generated. Primary assembly contigs were scaffolded with chromosome conformation Hi-C data. Manual assembly curation corrected 6 missing/misjoins, reducing the assembly length by $0.8 \%$ and the scaffold number by $13.5 \%$.

The final assembly has a total length of $325 \mathrm{Mb}$ in 33 sequence scaffolds with a scaffold $\mathrm{N} 50$ of $11 \mathrm{Mb}$ (Table 1). Of the assembly sequence, $100 \%$ was assigned to 32 chromosomal-level scaffolds, representing 30 autosomes (numbered by sequence length), and the $\mathrm{W}$ and $\mathrm{Z}$ sex chromosome (Figure 2-Figure 5; Table 2). The assembly has a BUSCO (Simão et al., 2015) v5.1.2 completeness of $99.0 \%$ (single $98.7 \%$, duplicated $0.3 \%$, fragmented $0.2 \%$, missing $0.8 \%$ ) using the lepidoptera_odb10 reference set. While not fully phased, the assembly deposited is of one haplotype. Contigs corresponding to the second haplotype have also been deposited.

\section{Gene annotation}

The Ensembl gene annotation system (Aken et al., 2016) was used to generate annotation for the Colias crocea assembly (GCA_905220415.1, Table 1). The annotation was created primarily through alignment of transcriptomic data to the genome, with gap filling via protein to-genome alignments of a select set of proteins from UniProt (UniProt Consortium, 2019) and OrthoDB (Kriventseva et al., 2008). Prediction tools, CPC2
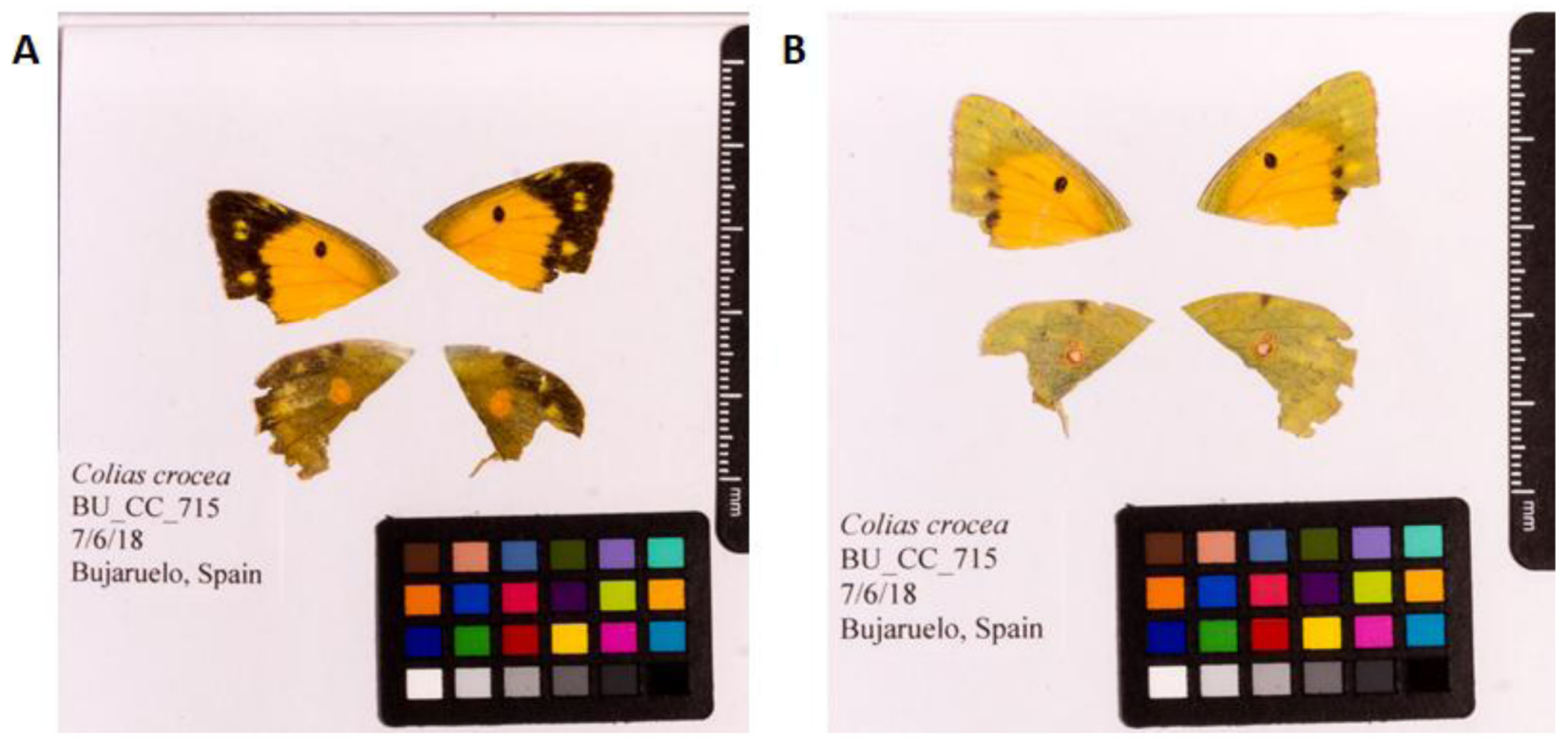

Figure 1. Fore and hind wings of Colias crocea specimen from which the genome was sequenced. (A) Dorsal surface view of wings from specimen BU_CC_715 (ilColCroc2) from Bujaruelo, Spain, used to generate Pacific Biosciences and 10X genomics data. (B) Ventral surface view of wings from specimen BU_CC_715 (ilColCroc2) from Bujaruelo, Spain, used to generate Pacific Biosciences and 10X genomics data. 


\section{Table 1. Genome data for Colias crocea, ilColCroc2.1.}

\begin{tabular}{|c|c|}
\hline \multicolumn{2}{|l|}{ Project accession data } \\
\hline Assembly identifier & ilColCroc2.1 \\
\hline Species & Colias crocea (also known as Colias croceus) \\
\hline Specimen & ilColCroc2 \\
\hline NCBI taxonomy ID & NCBI:txid72248 \\
\hline BioProject & PRJEB42878 \\
\hline BioSample ID & SAMEA7523360 \\
\hline Isolate information & Female, abdomen/thorax \\
\hline \multicolumn{2}{|l|}{ Raw data accessions } \\
\hline PacificBiosciences SEQUEL II & ERR6558184 \\
\hline 10X Genomics Illumina & ERR6054394-ERR6054397 \\
\hline Hi-C Illumina & ERR6054398 \\
\hline Illumina PolyA RNAseq & ERR6054399 \\
\hline \multicolumn{2}{|l|}{ Genome assembly } \\
\hline Assembly accession & GCA_905220415.1 \\
\hline Accession of alternate haplotype & GCA_905220445.1 \\
\hline Span (Mb) & 325 \\
\hline Number of contigs & 42 \\
\hline Contig N50 length (Mb) & 11 \\
\hline Number of scaffolds & 33 \\
\hline Scaffold N50 length (Mb) & 11 \\
\hline Longest scaffold (Mb) & 15 \\
\hline BUSCO* genome score & C:99.0\%[S:98.6\%,D:0.4\%],F:0.2\%,M:0.8\%,n:1658 \\
\hline \multicolumn{2}{|l|}{ Gene annotation } \\
\hline Number of protein-coding genes & 13,830 \\
\hline $\begin{array}{l}\text { Average length of protein coding } \\
\text { sequence (bp) }\end{array}$ & 1.631 \\
\hline $\begin{array}{l}\text { Average number of exons per } \\
\text { gene }\end{array}$ & 8 \\
\hline Average exon size (bp) & 359 \\
\hline Average intron size (bp) & 2,027 \\
\hline
\end{tabular}

(Kang et al., 2017) and RNAsamba (Camargo et al., 2020), were used to aid determination of protein coding genes.

\section{Methods}

Sample acquisition and nucleic acid extraction A female (ilColCroc2) and a male (ilColCroc3) C. crocea were collected from Bujaruelo, Aragon, Spain (latitude 42.7, longitude -0.1) by Sam Ebdon, Alex Macintosh (both University of Edinburgh), Alex Hayward and Karl Wotton (both University of Exeter). Samples were collected using a net and snapfrozen in liquid nitrogen.

DNA was extracted at the Wellcome Sanger Institute (WSI) Scientific Operations core from the thorax of ilColCroc2 using 


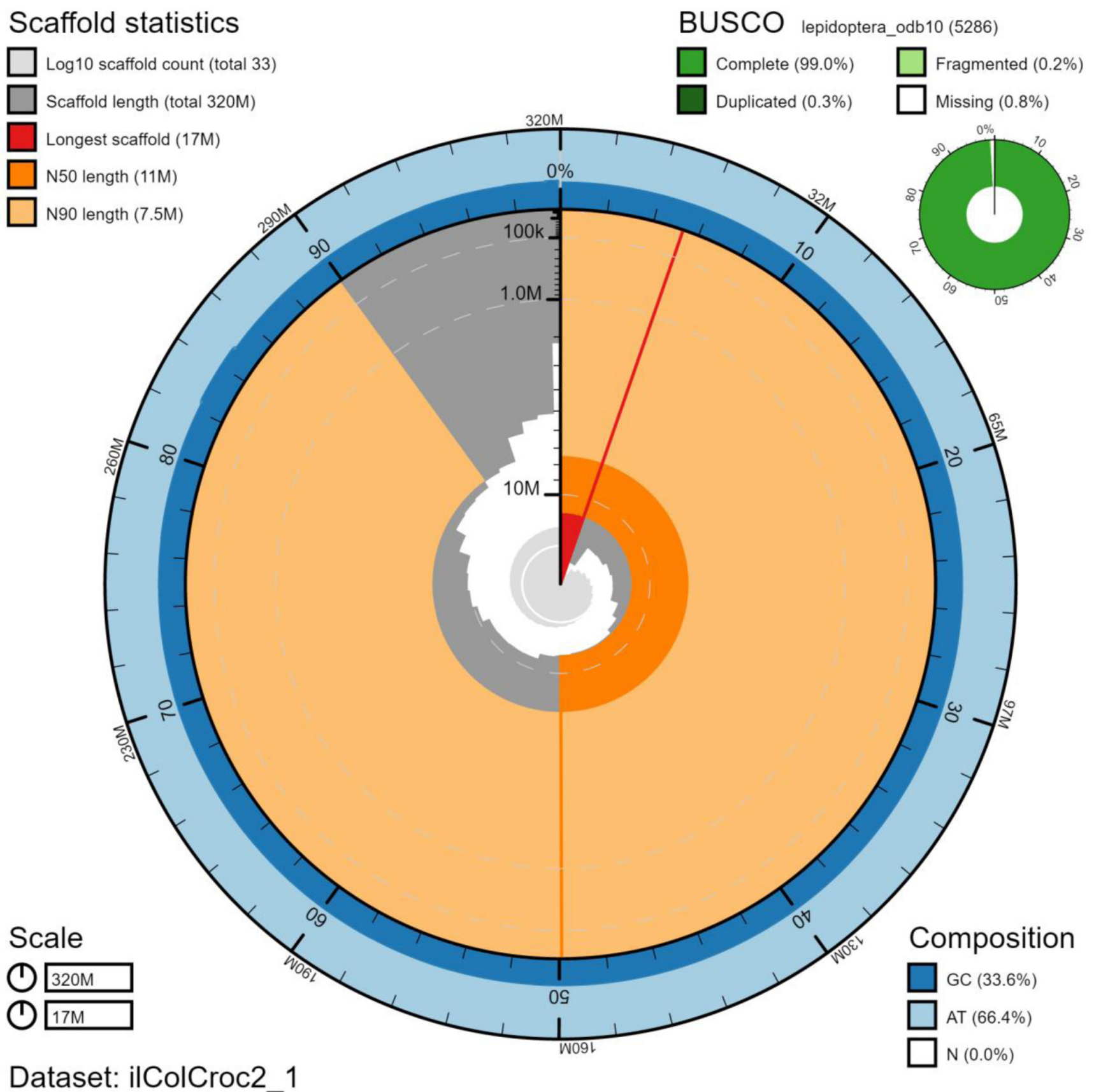

Figure 2. Genome assembly of Colias crocea, ilColCroc2.1: metrics. The BlobToolKit Snailplot shows N50 metrics and BUSCO gene completeness. The main plot is divided into 1,000 size-ordered bins around the circumference with each bin representing $0.1 \%$ of the $324,912,214$ bp assembly. The distribution of chromosome lengths is shown in dark grey with the plot radius scaled to the longest chromosome present in the assembly $(17,237,107 \mathrm{bp}$, shown in red). Orange and pale-orange arcs show the N50 and N90 chromosome lengths (11,204,669 and 7,474,634 bp), respectively. The pale grey spiral shows the cumulative chromosome count on a log scale with white scale lines showing successive orders of magnitude. The blue and pale-blue area around the outside of the plot shows the distribution of GC, AT and N percentages in the same bins as the inner plot. A summary of complete, fragmented, duplicated and missing BUSCO genes in the lepidoptera_odb10 set is shown in the top right. An interactive version of this figure is available at https://blobtoolkit.genomehubs. org/view/ilColCroc2.1/dataset/ilColCroc2_1/snail. 


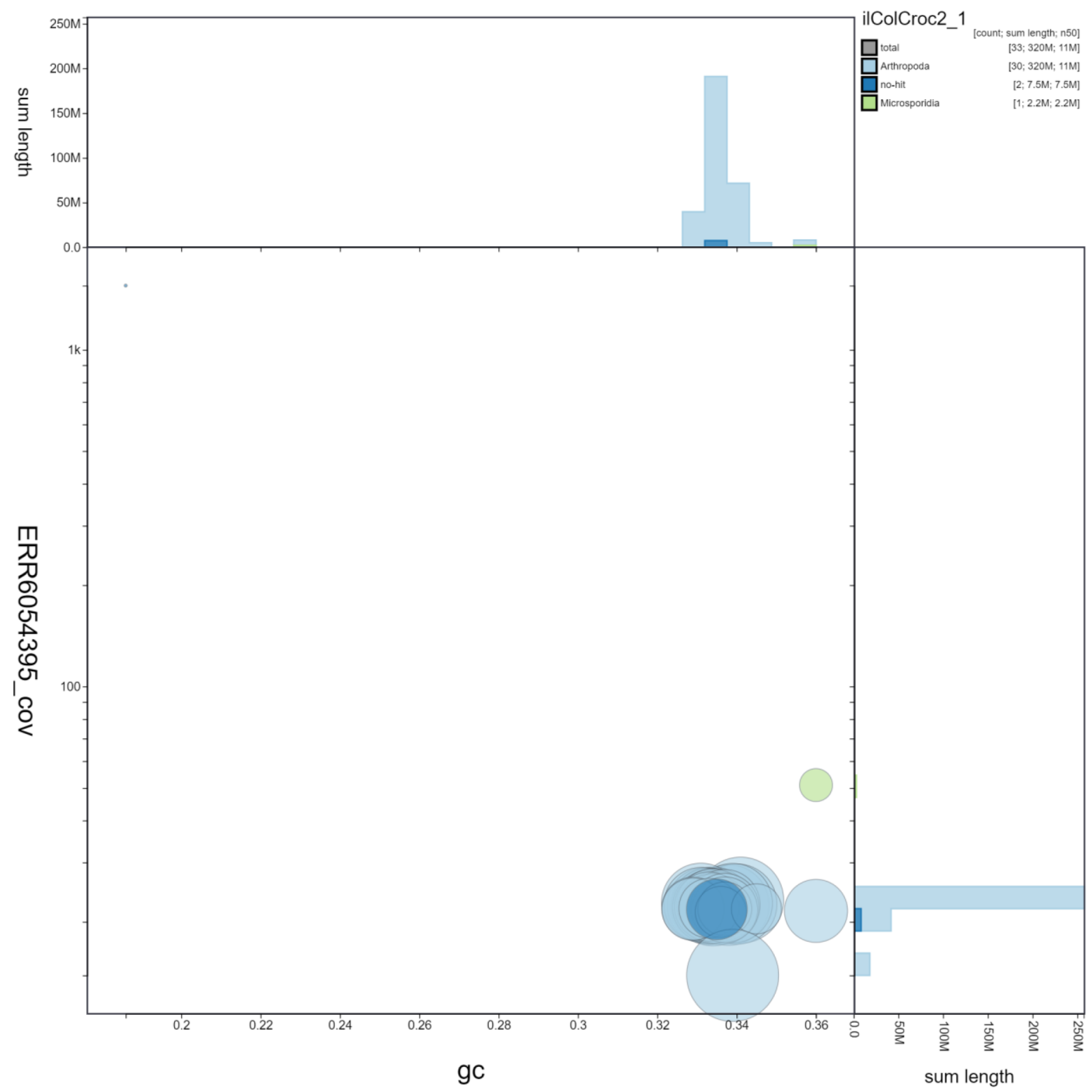

Figure 3. Genome assembly of Colias crocea, ilColCroc2.1: GC coverage. BlobToolKit GC-coverage plot. Chromosomes are coloured by phylum. Circles are sized in proportion to scaffold length. Histograms show the distribution of chromosome length sum along each axis. An interactive version of this figure is available at https://blobtoolkit.genomehubs.org/view/ilColCroc2.1/dataset/ilColCroc2_1/blob.

the Qiagen MagAttract HMW DNA kit, according to the manufacturer's instructions. RNA was extracted from the thorax of ilColCroc3 in the Tree of Life Laboratory at the WSI using TRIzol (Invitrogen), according to the manufacturer's instructions. RNA was then eluted in $50 \mu \mathrm{l}$ RNAse-free water and its concentration RNA assessed using a Nanodrop spectrophotometer and Qubit Fluorometer using the Qubit RNA Broad-Range (BR)
Assay kit. Analysis of the integrity of the RNA was done using Agilent RNA 6000 Pico Kit and Eukaryotic Total RNA assay.

\section{Sequencing}

Pacific Biosciences HiFi circular consensus and 10X Genomics read cloud sequencing libraries were constructed according 


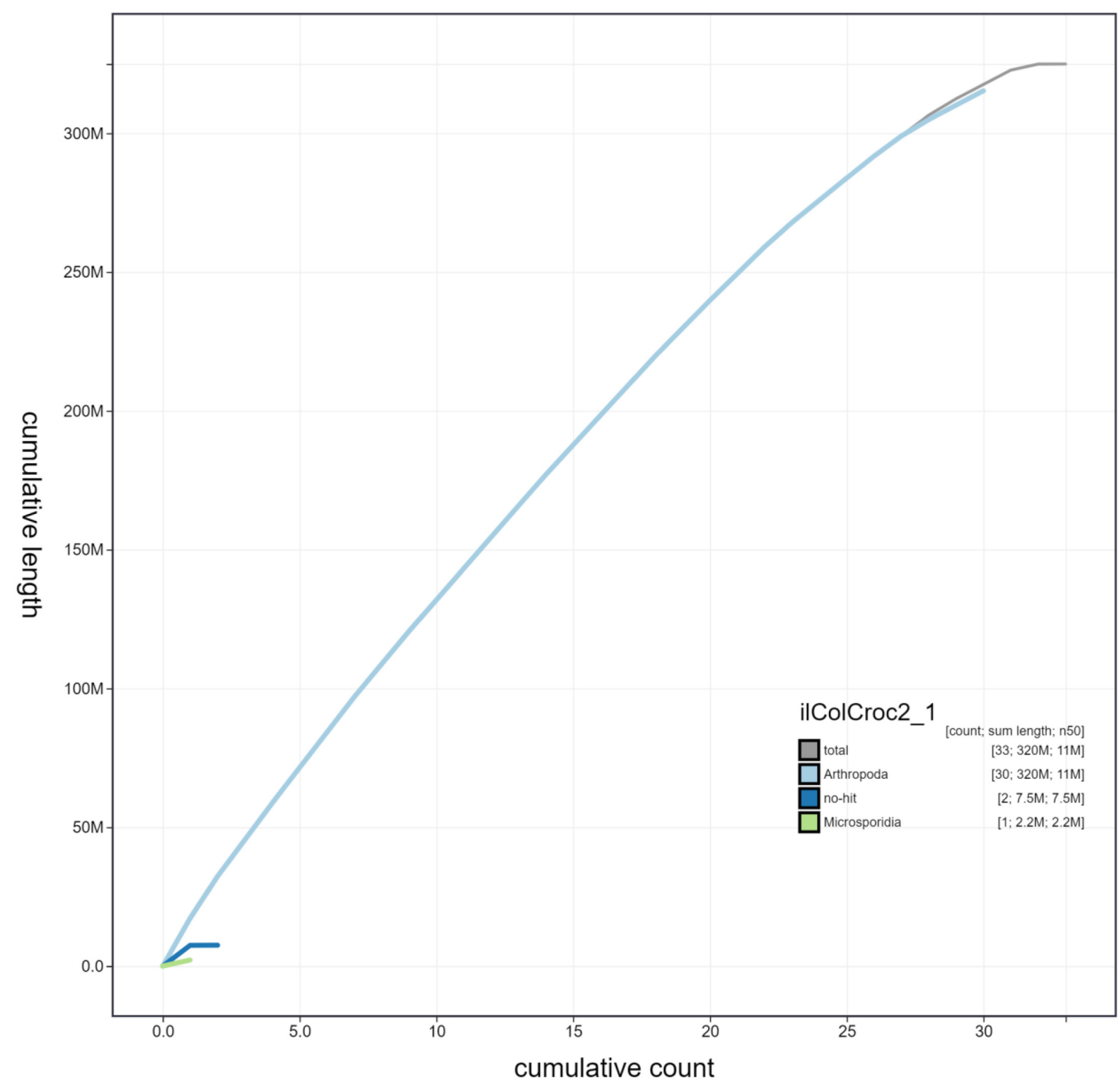

Figure 4. Genome assembly of Colias crocea, ilColCroc2.1: cumulative sequence. BlobToolKit cumulative sequence plot. The grey line shows cumulative length for all chromosomes. Coloured lines show cumulative lengths of chromosomes assigned to each phylum using the buscogenes taxrule. An interactive version of this figure is available at https://blobtoolkit.genomehubs.org/view/ilColCroc2.1/dataset/ ilColCroc2_1/cumulative.

to the manufacturers' instructions. SPoly(A) RNA-Seq libraries were constructed using the NEB Ultra II RNA Library Prep kit. Sequencing was performed by the Scientific Operations core at the Wellcome Sanger Institute on Pacific Biosciences
SEQUEL II (HiFi), Illumina HiSeq X (10X) and Illumina HiSeq 4000 (RNA-Seq) instruments. Hi-C data were generated from abdomen tissue of ilColCroc2 using the Arima v2.0 kit and sequenced on HiSeq X. 


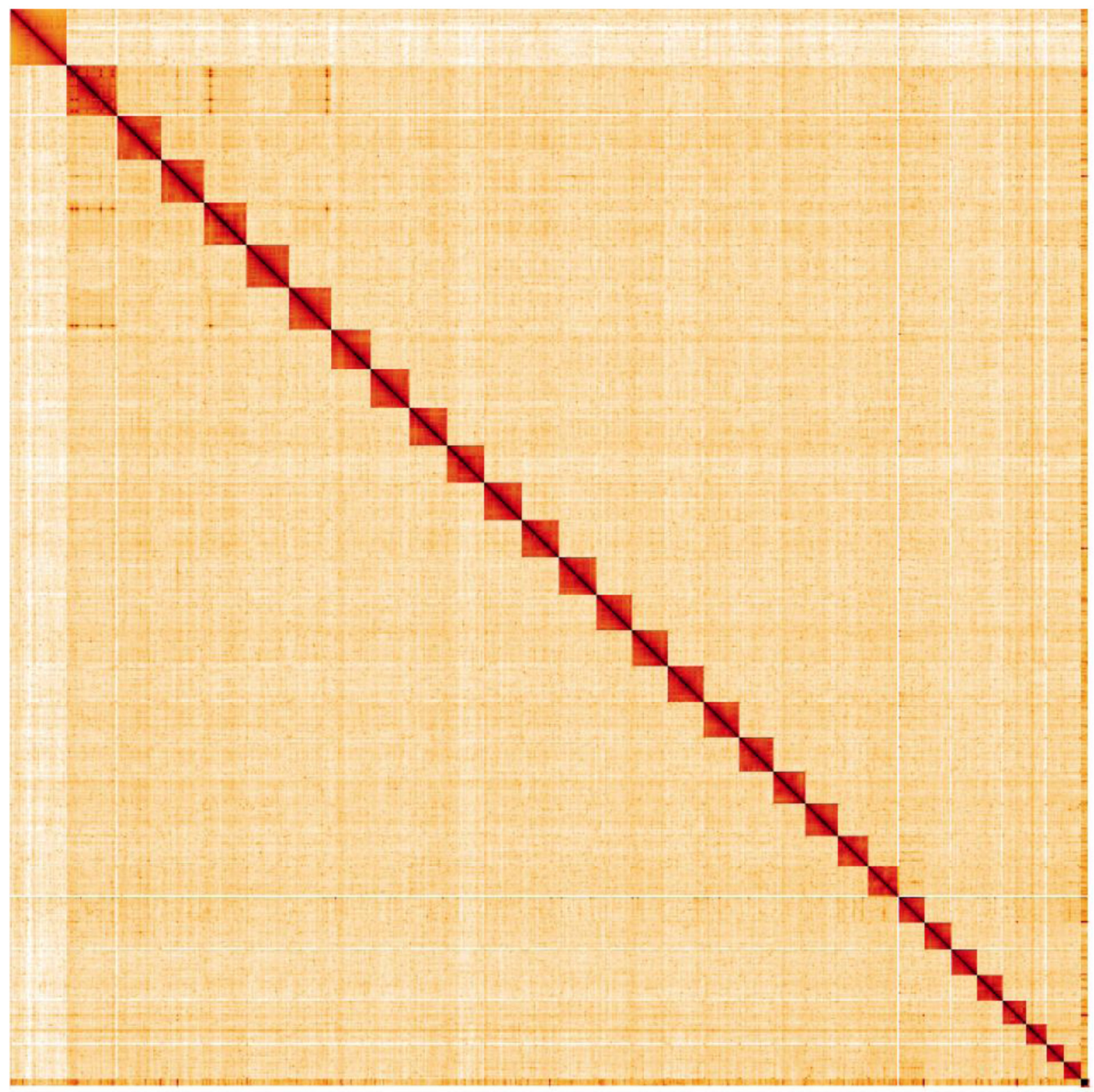

Figure 5. Genome assembly of Colias crocea, ilColCroc2.1: Hi-C contact map. Hi-C contact map of the ilColCroc2.1 assembly, visualised in HiGlass.

\section{Genome assembly}

Assembly was carried out with Hifiasm (Cheng et al., 2021). Haplotypic duplication was identified and removed with purge_dups (Guan et al., 2020). One round of polishing was performed by aligning $10 \mathrm{X}$ Genomics read data to the assembly with longranger align, calling variants with freebayes (Garrison \& Marth, 2012). The assembly was then scaffolded with Hi-C data (Rao et al., 2014) using SALSA2 (Ghurye et al., 2019). The assembly was checked for contamination and corrected using the gEVAL system (Chow et al., 2016) as described previously (Howe et al., 2021). Manual curation was performed using gEVAL, HiGlass (Kerpedjiev et al., 2018) and Pretext. The mitochondrial genome was assembled using MitoHiFi (Uliano-Silva et al., 2021). The genome was analysed and BUSCO scores generated within the BlobToolKit environment (Challis et al., 2020). Table 3 contains a list of all software tool versions used, where appropriate.

\section{Ethical/compliance issues}

The materials that have contributed to this genome note were supplied by a Tree of Life collaborator. The Wellcome Sanger Institute employs a process whereby due diligence is carried 
Table 2. Chromosomal pseudomolecules in the genome assembly of Colias crocea, ilColCroc2.1.

\begin{tabular}{|c|c|c|c|}
\hline INSDC accession & Chromosome & Size (Mb) & GC\% \\
\hline HG991959.1 & 1 & 15.09 & 34.1 \\
\hline HG991960.1 & 2 & 13.25 & 33.8 \\
\hline HG991961.1 & 3 & 13.17 & 34 \\
\hline HG991962.1 & 4 & 12.85 & 33.9 \\
\hline HG991963.1 & 5 & 12.69 & 33.4 \\
\hline HG991964.1 & 6 & 12.66 & 33.1 \\
\hline HG991965.1 & 7 & 12.04 & 33.3 \\
\hline HG991966.1 & 8 & 11.65 & 33.2 \\
\hline HG991967.1 & 9 & 11.34 & 33.4 \\
\hline HG991968.1 & 10 & 11.32 & 33.3 \\
\hline HG991969.1 & 11 & 11.31 & 33.4 \\
\hline HG991970.1 & 12 & 11.20 & 33.4 \\
\hline HG991971.1 & 13 & 11.13 & 33.1 \\
\hline HG991972.1 & 14 & 10.75 & 33.3 \\
\hline HG991973.1 & 15 & 10.73 & 33.5 \\
\hline HG991974.1 & 16 & 10.69 & 33.4 \\
\hline HG991975.1 & 17 & 10.66 & 33.6 \\
\hline HG991976.1 & 18 & 10.16 & 33.7 \\
\hline HG991977.1 & 19 & 9.83 & 33.4 \\
\hline HG991978.1 & 20 & 9.70 & 33.3 \\
\hline HG991979.1 & 21 & 9.58 & 33.7 \\
\hline HG991980.1 & 22 & 8.79 & 33.7 \\
\hline HG991981.1 & 23 & 8.06 & 36 \\
\hline HG991982.1 & 24 & 7.97 & 32.9 \\
\hline HG991983.1 & 25 & 7.88 & 32.9 \\
\hline HG991984.1 & 26 & 7.47 & 33.5 \\
\hline HG991985.1 & 27 & 7.27 & 33.3 \\
\hline HG991986.1 & 28 & 5.88 & 33.7 \\
\hline HG991987.1 & 29 & 5.28 & 33.6 \\
\hline HG991988.1 & 30 & 5.08 & 34.5 \\
\hline HG991989.1 & W & 2.16 & 36 \\
\hline HG991958.1 & Z & 17.24 & 33.9 \\
\hline HG991990.1 & MT & 0.02 & 18.7 \\
\hline
\end{tabular}

\section{Table 3. Software tools used.}

\begin{tabular}{|c|c|c|}
\hline Software tool & Version & Source \\
\hline Hifiasm & 0.12 & Cheng et al., 2021 \\
\hline purge_dups & 1.2 .3 & Guan et al., 2020 \\
\hline SALSA2 & 2.2 & Ghurye et al., 2019 \\
\hline longranger align & 2.2 .2 & $\begin{array}{l}\text { https:// } \\
\text { support.10xgenomics. } \\
\text { com/genome-exome/ } \\
\text { software/pipelines/latest/ } \\
\text { advanced/other-pipelines }\end{array}$ \\
\hline freebayes & 1.3.1-17-gaa2ace8 & Garrison \& Marth, 2012 \\
\hline MitoHiFi & 1.0 & $\begin{array}{l}\text { https://github.com/ } \\
\text { marcelauliano/MitoHiFi }\end{array}$ \\
\hline gEVAL & N/A & Chow et al., 2016 \\
\hline HiGlass & 1.11 .6 & Kerpedjiev et al., 2018 \\
\hline PretextView & $0.1 . x$ & $\begin{array}{l}\text { https://github.com/wtsi- } \\
\text { hpag/PretextView }\end{array}$ \\
\hline BlobToolKit & 2.6 .2 & Challis et al., 2020 \\
\hline
\end{tabular}

out proportionate to the nature of the materials themselves, and the circumstances under which they have been/are to be collected and provided for use. The purpose of this is to address and mitigate any potential legal and/or ethical implications of receipt and use of the materials as part of the research project, and to ensure that in doing so we align with best practice wherever possible.

The overarching areas of consideration are:

- Ethical review of provenance and sourcing of the material;

- Legality of collection, transfer and use (national and international).

Each transfer of samples is undertaken according to a Research Collaboration Agreement or Material Transfer Agreement entered into by the Tree of Life collaborator, Genome Research Limited (operating as the Wellcome Sanger Institute) and in some circumstances other Tree of Life collaborators.

\section{Data availability}

European Nucleotide Archive: Colias croceus (clouded yellow). Accession number PRJEB42949; https://identifiers.org/ena.embl/ PRJEB42949.

The genome sequence is released openly for reuse. The C. crocea genome sequencing initiative is part of the Darwin Tree of Life (DToL) project. All raw sequence data and the assembly have been deposited in INSDC databases. Raw data and assembly accession identifiers are reported in Table 1. 


\section{Author information}

Members of the Darwin Tree of Life Barcoding collective are listed here: https://doi.org/10.5281/zenodo.4893704.

Members of the Wellcome Sanger Institute Tree of Life programme collective are listed here: https://doi.org/10.5281/ zenodo.5377053.
Members of Wellcome Sanger Institute Scientific Operations: DNA Pipelines collective are listed here: https://doi.org/10.5281/ zenodo.4790456.

Members of the Tree of Life Core Informatics collective are listed here: https://doi.org/10.5281/zenodo.5013542.

Members of the Darwin Tree of Life Consortium are listed here: https://doi.org/10.5281/zenodo.4783559.
Aken BL, Ayling S, Barrell D, et al.: The Ensembl Gene Annotation System. Database (Oxford). 2016; 2016: baw093.

PubMed Abstract | Publisher Full Text | Free Full Text

Camargo AP, Sourkov V, Pereira GAG, et al.: RNAsamba: Neural NetworkBased Assessment of the Protein-Coding Potential of RNA Sequences. NAR Genom Bioinform. 2020; 2(1): lqz024.

PubMed Abstract | Publisher Full Text | Free Full Text

Challis R, Richards E, Rajan J, et al.: BlobToolKit - Interactive Quality Assessment of Genome Assemblies. G3 (Bethesda). 2020; 10(4): 1361-74. PubMed Abstract | Publisher Full Text | Free Full Text

Cheng $\mathrm{H}$, Concepcion GT, Feng X, et al:: Haplotype-Resolved de Novo Assembly Using Phased Assembly Graphs with Hifiasm. Nat Methods. 2021 18(2): 170-75.

PubMed Abstract | Publisher Full Text | Free Full Text

Chow W, Brugger K, Caccamo M, et al.: gEVAL - a Web-Based Browser for Evaluating Genome Assemblies. Bioinformatics. 2016; 32(16): 2508-10. PubMed Abstract | Publisher Full Text | Free Full Text

Fox R, Brereton TM, Asher J, et al.: The State of the UK's Butterflies 2015. 2015 .

Reference Source

Garrison E, Marth G: Haplotype-Based Variant Detection from Short-Read Sequencing. arXiv: 1207.3907. 2012.

Reference Source

Ghurye J, Rhie A, Walenz BP, et al.: Integrating Hi-C Links with Assembly Graphs for Chromosome-Scale Assembly. PLoS Comput Biol. 2019; 15(8): e1007273.

PubMed Abstract | Publisher Full Text | Free Full Text

Guan D, McCarthy SA, Wood J, et al.: Identifying and Removing Haplotypic Duplication in Primary Genome Assemblies. Bioinformatics. 2020; 36(9): 2896-98.

PubMed Abstract | Publisher Full Text | Free Full Text

Howe K, Chow W, Collins J, et al.: Significantly Improving the Quality of Genome Assemblies through Curation. GigaScience. 2021; 10(1): giaa153. PubMed Abstract | Publisher Full Text | Free Full Text
Kang Y], Yang DC, Kong L, et al.: CPC2: A Fast and Accurate Coding Potential Calculator Based on Sequence Intrinsic Features. Nucleic Acids Res. 2017; 45(W1): W12-16.

PubMed Abstract | Publisher Full Text | Free Full Text

Kerpedjiev P, Abdennur N, Lekschas F, et al: HiGlass: Web-Based Visual Exploration and Analysis of Genome Interaction Maps. Genome Biol. 2018; 19(1): 125.

PubMed Abstract | Publisher Full Text | Free Full Text

Kriventseva EV, Rahman N, Espinosa O, et al: OrthoDB: The Hierarchical Catalog of Eukaryotic Orthologs. Nucleic Acids Res. 2008; 36(Database issue): D271-75.

PubMed Abstract | Publisher Full Text | Free Full Text

Rao SSP, Huntley MH, Durand NC, et al:: A 3D Map of the Human Genome at Kilobase Resolution Reveals Principles of Chromatin Looping. Cell. 2014; 159(7): 1665-80.

PubMed Abstract | Publisher Full Text | Free Full Text

Simão FA, Waterhouse RM, Ioannidis P, et al.: BUSCO: Assessing Genome Assembly and Annotation Completeness with Single-Copy Orthologs. Bioinformatics. 2015; 31(19): 3210-12.

PubMed Abstract | Publisher Full Text

Uliano-Silva M, Nunes JGF, Krasheninnikova K, et al.: marcelauliano/MitoHiFi: mitohifi_v2.0. 2021

Publisher Full Text

UniProt Consortium: UniProt: A Worldwide Hub of Protein Knowledge.

Nucleic Acids Res. 2019; 47(D1): D506-15.

PubMed Abstract | Publisher Full Text | Free Full Text

van Swaay C, Verovnik WI, Wiemers R, et al: IUCN Red List of Threatened

Species: Colias Crocea. IUCN Red List of Threatened Species. 2010

Reference Source

Woronik A, Tunström K, Perry MW, et al: A Transposable Element Insertion Is Associated with an Alternative Life History Strategy. Nat Commun. 2019; 10(1): 5757.

PubMed Abstract | Publisher Full Text | Free Full Text 


\section{Open Peer Review}

\section{Current Peer Review Status:}

\section{Version 1}

Reviewer Report 20 September 2022

https://doi.org/10.21956/wellcomeopenres.19119.r52189

(C) 2022 Steward R. This is an open access peer review report distributed under the terms of the Creative Commons Attribution License, which permits unrestricted use, distribution, and reproduction in any medium, provided the original work is properly cited.

\section{Rachel A Steward}

Department of Zoology, Stockholm University, Stockholm, Sweden

The authors present a highly contiguous and complete genome assembly for the Pierid butterfly, Colias crocea. The existence of a female-limited color polymorphism, in which females are either white or orange, has made this and other species in the genus emerging model systems for the study of alternative life history strategies and the maintenance of ancient polymorphisms.

For this genome assembly, the authors used a combination of sequence data from an orange female, providing detailed information about the sequencing and assembly steps. The authors also used RNAseq data sampled from the thorax of a single adult male to generate an annotation for this assembly, which may limit its scope for describing both the protein coding and noncoding features of this assembly. Overall, I agree with the previous reviewer that a genome assembly of this quality is highly beneficial (and has likely already been useful) for the study of $C$. crocea and for comparative research in butterfly genomics.

\section{Critiques:}

I support the critiques of the first reviewer: (1) that more details should be provided about the parameters and options for software used to generate both the assembly and the annotation; and (2) that the manuscript would benefit from a more detailed explanation of the differences between this assembly and existing genomic resources.

Details are lacking about the annotation. Given the annotation was made using RNA from a single male thorax, it is possible that there were many important coding and noncoding transcripts missing in the sample. The protein prediction tools subsequently used to improve the annotation may have filled these gaps, but I think it is important that the authors both acknowledge these limitations and include some evaluation of annotation quality. The authors could run BUSCO on the protein set or could compare orthologs between this and other well-annotated Lepidopteran gene sets. Also, summary statistics (e.g., average length of protein coding sequence) provided in Table 1 should include some measure of variation (95\% CI, etc.) 


\title{
Is the rationale for creating the dataset(s) clearly described?
}

Yes

Are the protocols appropriate and is the work technically sound?

Yes

Are sufficient details of methods and materials provided to allow replication by others? Partly

Are the datasets clearly presented in a useable and accessible format?

Yes

Competing Interests: No competing interests were disclosed.

Reviewer Expertise: Evolutionary ecology, gene expression, alternative splicing

I confirm that I have read this submission and believe that I have an appropriate level of expertise to confirm that it is of an acceptable scientific standard.

Reviewer Report 22 November 2021

https://doi.org/10.21956/wellcomeopenres.19119.r46607

(C) 2021 Woronik A. This is an open access peer review report distributed under the terms of the Creative Commons Attribution License, which permits unrestricted use, distribution, and reproduction in any medium, provided the original work is properly cited.

\author{
Alyssa Woronik \\ Sacred Heart University, Fairfield, CT, USA
}

\section{Summary of the article and contributions:}

The authors generate a high quality genome for the butterfly Colias crocea using PacBio, 10X Genomics, and $\mathrm{HiC}$ sequencing data. These sequencing datasets were generated from an orange C. crocea female. The resulting genome was annotated using RNA-Seq data from a male C. crocea. This is a high quality genome that is more contiguous and complete than a previously published $C$. crocea genome (Woronik et al., 2019 ${ }^{1}$ ). Because this butterfly species harbors structural variation that results in an alternative life history strategy, such high quality genomes are necessary for in depth study of these (and potentially other) important genomic regions. Overall, this genome will contribute to the understanding of this butterfly's biology and ecology.

\section{Critiques:}

1. The authors reported the software and versions used for their assembly. However, I could not find a place where the various options used in those pipelines were stated. Would it be possible for the authors to include the options used in the assembly and annotation commands? This would increase the reproducibility of this work. This could be done by adding another column to Table 3 "Options used" or by including their scripts as a 
supplemental file.

2. The authors point out that another high quality genome exists for $C$. crocea. This genome was generated from Alba females and annotated using RNA-Seq data from the wings and abdomens of several female pupas. Adding a sentence to the "Background" that highlights the differences between the datasets could be useful for readers interested in using this data as they complement each other nicely.

\section{References}

1. Woronik A, Tunström K, Perry M, Neethiraj R, et al.: A transposable element insertion is associated with an alternative life history strategy. Nature Communications. 2019; 10 (1). Publisher Full Text

Is the rationale for creating the dataset(s) clearly described?

Yes

Are the protocols appropriate and is the work technically sound?

Yes

Are sufficient details of methods and materials provided to allow replication by others? Partly

Are the datasets clearly presented in a useable and accessible format? Yes

Competing Interests: No competing interests were disclosed.

Reviewer Expertise: Evolutionary genomics and developmental genetics

I confirm that I have read this submission and believe that I have an appropriate level of expertise to confirm that it is of an acceptable scientific standard. 